\title{
Hippocampal lesions produce a temporally graded retrograde amnesia on a dry version of the Morris swimming task
}

\author{
JOHN L. KUBIE \\ State University of New York, Brooklyn, New York \\ ROBERT J. SUTHERLAND \\ University of New Mexico, Albuquerque, New Mexico \\ and \\ ROBERT U. MULLER \\ State University of New York, Brooklyn, New York
}

\begin{abstract}
Using a dry version of the Morris hidden-goal swimming task, we reexplored the retrograde and anterograde effects of hippocampal lesions on spatial performance in rats. Rats were trained to find food buried under sawdust in a 183-cm-diameter gray cylinder; the location of the buried food was constant for each rat. Either 1 week or 14 weeks after training the rats were subjected to massive hippocampal + dentate lesions by multiple injections of neurotoxic agents. Rats with a 1-week training-lesion interval could not reacquire the ability to get to the hidden food, although they performed normally when the goal location was marked with a dowel. In contrast, rats with a 14-week training-lesion interval relearned finding the buried food at a nearly normal rate. The two groups together suggest that memory for the buried food task is susceptible to graded retrograde amnesia in a fashion similar to explicit memory in humans. Additional testing of the 14-week training-lesion group revealed, however, that the consolidated memory was not fully formed; these rats were incapable of learning to go to a new location in the same cylinder, although sham lesioned rats readily transferred. To explain this pattern of results, we propose that (1) rats with an intact hippocampus have available a neural map of the environment that permits them to learn to go from anywhere to anywhere else, and (2) hippocampally lesioned rats have no such map, but are able to show some navigational skills by using a vector field representation of the environment that permits them to get from anywhere to a single location. In a separate experiment, we provide support for the notion that the rodent hippocampus has a specialized role in spatial memory by showing that hippocampally lesioned rats performed normally in a visual object recognition task whose motor and motivational components were the same as in the buried food task.
\end{abstract}

In humans, lesions of the hippocampus produce deficits in "explicit" memory (also called episodic memory), but spare "implicit" memory. Explicit memory is for specific facts about the world; it is memory for autobiographical events, geographical knowledge, and so on. In contrast, implicit memory is for the methods necessary to accomplish specific tasks. Thus, it is possible for a person with hippocampal lesions to know how to solve a certain problem (e.g., mirror tracing) but to not know that he/she is able to solve the problem (Corkin, 1965, 1968).

The amnesia for explicit information caused by hippocampal damage has two components, anterograde and retrograde. Anterograde amnesia is the loss of the ability

This work was supported by N.I.H. Grant 20686 to J.L.K. and R.U.M. We thank Shahrokh Dayyani for help in all phases of the study. Correspondence should be addressed to J. L. Kubie, Department of Anatomy and Cell Biology, Box 5, SUNY Health Science Center at Brooklyn, 450 Clarkson Ave., Brooklyn, NY 11203 (e-mail: jkubie@netmail. hscbklyn.edu). to acquire new explicit information; it is an inability to remember new events. Retrograde amnesia is the loss of memories for events and facts acquired before hippocampal damage. Retrograde amnesia might be ungraded, which means that memory is lost for almost all events that occurred before the lesions. It might instead be graded, such that all memory is lost for events that happened just before the lesion but progressively less memory is lost for events that preceded the damage by progressively greater intervals. Empirically, whether retrograde amnesia is graded or not depends on both the extent of the hippocampal damage and the exact nature of the explicit information (Nadel \& Moscovitch, 1997; see also Squire \& Alvarez, 1995).

The most widely accepted explanation of the hippocampal amnesic syndrome depends on the idea of consolidation, which is the process by which recent memories that depend on an intact hippocampus come, with the passage of time, to be resistant to hippocampal damage. In this view, recently summarized by Squire and Alvarez 
(1995), the hippocampus acts as a temporary store for explicit memory. Initially, the hippocampus rapidly registers information about an event in cooperation with neocortex. Subsequently, information about the event is transformed so that the neocortex, by itself, can store the information and the hippocampus is no longer necessary. According to the consolidation model, anterograde amnesia results because the required temporary memory store in the hippocampus is unavailable. Similarly, retrograde amnesia results because certain memories did not exist long enough before the lesion for the neocortical trace to become stable. Finally, the grading of retrograde amnesia reflects the time course of consolidation. The slow kinetics of consolidation may reflect a way of preventing instability in the cortical trace (McClelland, McNaughton, \& O'Reilly, 1995).

An alternative picture of the amnesic syndrome focuses on cases in which retrograde amnesia extends for several decades and may occupy virtually the entire premorbid lifespan of the individual. Nadel and Moscovitch (1997) have argued that the notion of consolidation becomes suspect if retrograde amnesia can be ungraded. How, then, can graded retrograde amnesia be explained without appealing to consolidation? Nadel and Moscovitch began with the accepted notions that the content of explicit memory is always stored in neocortex and that the content must always be accessed through "index" or "pointer" information stored in the hippocampus. They then proposed that each time the neocortical content of a memory is recalled, it is recalled in a new context, and that each recall generates an additional pointer to the content. The number of pointers to the neocortical information therefore grows with the number of times the content is recalled. Since recalls are distributed over time, the number of pointers to old explicit information should be larger than the number of pointers to new explicit information. They also argued that old memories are more resistant to hippocampal damage because a certain amount of damage may remove only a fraction of the accumulated pointers, whereas the same damage may remove all the pointers to new memories. Finally, they argued that certain types of memories that depend on special hippocampal functions will be affected regardless of the interval between learning and lesion and will therefore always show a flat retrograde deficit.

Thus, the ability to distinguish between two very different explanations of the amnesic syndrome depends critically on whether retrograde amnesia is graded or flat after very extensive or complete destruction of the hippocampus. The requirement for precise anatomical lesions, however, makes it extremely difficult to resolve this issue by testing human patients since it is highly unlikely that complete destruction of the hippocampus with no damage to other structures will occur by chance. It is therefore clear that an animal model is required. Certainly a primate model would be of great value, but presently there is no task for monkeys that appears to both use explicit memory and that is specifically and undisputedly impaired by hippocampal lesions (Meunier, Bachevalier, Mishkin, \& Murray, 1993; Meunier, Hadfield, Bachevalier, \& Murray, 1996). Fortunately, an alternative exists in the form of spatial memory in rodents that is clearly impaired by hippocampal damage; rats with hippocampal lesions show anterograde amnesia in several spatial navigation tasks (Morris, Garrud, Rawlins, \& O'Keefe, 1982; Morris, Schenk, Tweedie, \& Jarrard, 1990; Sutherland, Whishaw, \& Kolb, 1983; Winocur, 1990).

There have been several attempts using the Morris swimming task to determine whether spatial retrograde amnesia in rats is graded or flat. Dentate gyrus lesions made with colchicine produced a temporally graded retrograde amnesia that was severe at 1 - and 4-week training-lesion intervals, but milder at 8- and 12-week intervals (Sutherland, Arnold, \& Rodriguez, 1987). In contrast, much more extensive hippocampal damage using kainic acid + colchicine injections caused severe retrograde amnesia at all training-lesion intervals back to 32 weeks (Astur, Mumby, Weisend, \& Sutherland, 1994). A similar severe, temporally independent retrograde amnesia extending back 14 weeks was reported by the Oxford group using ibotenic acid lesions in rats (Bolhuis, Stewart, \& Forrest, 1994). In contrast, Morris et al. (1990) found little if any retrograde impairment of performance with a 1 -week interval between the end of training and ibotenate lesions. Kim and Fanselow (1992) found graded retrograde amnesia in contextual fear conditioning. Thus, the picture with regard to a possible retrograde gradient is far from clear and deserves additional investigation.

One purpose of this paper is therefore to present a fresh look at the nature of the retrograde gradient in a spatial memory task. Using a new hidden-goal task tailored after the Morris task, we found clear evidence of graded retrograde amnesia. Specifically, if hippocampal lesions are made 1 week after a rat is trained to dig for buried food in a certain location, the retrograde effect is profound; with a l-week training-lesion interval, rats cannot relearn to dig at the hidden goal. In contrast, if hippocampal lesions are made 14 weeks after the same training, lesioned rats show almost the same retention of the goal location as sham-operated animals. Memory for the original goal location has therefore become independent of the integrity of the hippocampus. This result strongly supports the consolidation model (Squire \& Alvarez, 1995).

It is well known that rats trained to swim directly to a fixed hidden platform location in the water maze can quickly switch to swim directly to a different hidden platform location (Whishaw, Rod, \& Auer, 1994). Having found that a 14-week training-lesion interval spares the ability to get to the original buried food location, we next tested rats for their ability to learn to go to a new hidden location in the same "dry water maze." As from water maze results, rats with sham lesions quickly learned to 
get to a new buried food location. In contrast, lesioned rats were unable to learn to go to the new buried food location. Thus, the learning about the buried food task is incompletely preserved after hippocampal lesions; the retained knowledge does not include the ability to transfer to a new goal location. In line with the arguments of Nadel and Moscovitch (1997), there are aspects of spatial memory that require an intact hippocampus, regardless of the time of the original learning.

How can the specific ability to get to a previously learned goal be preserved even though the general ability to find a hidden goal location is devastated? We take the following position: Lesions of the hippocampus do not merely cause deficiencies in memory but also remove an expert navigational system-a neural network whose "place cells" confer on it many features of a cognitive map (Muller, 1996; O'Keefe \& Nadel, 1978). In short, it appears that the ability to get from any place in the dry maze to a specific goal location does not absolutely require this hippocampal map, whereas the ability to get from any place to an arbitrary goal location depends on an intact map. Thus, the spared memories of an environment in rats with hippocampal damage may be fundamentally different from the memory+computation possible for intact rats. Implications of this finding for understanding explicit memory in humans are explored in the General Discussion section.

Finally, in addition to investigating retrograde amnesia in a spatial memory task, we looked for retrograde amnesia in a visual object discrimination task that required the rat to find food buried under 1 of 10 small objects. We found that rats with a 2-week training-lesion interval were unimpaired in this task, demonstrating that the amnesia produced by hippocampal lesions in rats is not global. The preservation of object discrimination at short training-lesion intervals reinforces the idea that the hippocampus is involved in specific memories and computations and is not responsible in a global way for explicitlike memories and abilities.

We report the results of three experiments. Two experiments were concerned with the ability of rats with massive lesions of the hippocampal formation to solve a spatial problem similar to the Morris water maze (Morris, 1984). This "dry maze" was originally developed to make it easier to do single-cell recordings during navigation to a hidden goal than would be possible during swimming (Kubie, Muller, Dayyani, \& Morgenstern, 1987). In the version of the task used here, rats are taught to dig for food buried under a layer of sawdust at a fixed location relative to a single "polarizing cue" on the apparatus wall.

The third experiment dealt with the ability of hippocampally damaged rats to solve an object recognition task. The main purpose of this experiment was to test whether hippocampal lesions specifically disrupt the ability to learn spatial tasks or whether they produce more global learning deficits. Each experiment was done on a different group of rats, and the experiments were done at different times.

\section{METHOD}

\section{Subjects}

The experimental subjects in all experiments were young adult male Long-Evans rats (Blue Spruce Farms, Indianapolis, IN). The rats weighed about $250 \mathrm{~g}$ upon arrival and were fed ad lib until their weight stabilized. They were then food deprived until their weight fell to $90 \%$ of the ad-lib level. Thereafter, they were fed $12-15 \mathrm{~g}$ of rat pellets per day in addition to food rewards obtained during behavioral training and testing. Prior to any training, each rat was handled once per day for 5 days. During each day, the rat was put into the selected behavioral apparatus after several grams of Coco Puff cereal pieces were scattered onto the sawdust on the apparatus floor. Following this acclimatization, the rats were ready for "pretraining" in one of two apparati.

\section{Method for Navigational Experiments}

Apparatus. The apparatus for navigational experiments was a gray wooden cylinder $183 \mathrm{~cm}$ in diameter and $91.5 \mathrm{~cm}$ high. The cylinder was surrounded by a cylindrical curtain $244 \mathrm{~cm}$ in diameter that ran from the laboratory ceiling to below the edge of the cylinder, creating a controlled cue environment. The only intentionally introduced asymmetry in the environment was a rectangular white cardboard sheet bent to cover $90^{\circ}$ of the cylinder wall. The controlled-cue environment was lit by four overhead 24-W light bulbs arranged on the corners of a square. The interior of the cylinder was filled to a depth of $10 \mathrm{~cm}$ with sawdust that was replaced once per week. Each time the sawdust was replaced, $1,000 \mathrm{ml}$ of powdered Coco Puff cereal was mixed in to mask odors that might have emanated from the food dish.

The rats' behavior was monitored with an overhead TV camera. With this provision, it was possible for the experimenter to rapidly intervene when a rat found food or when the time limit for finding food was exceeded. The TV signal was also tape-recorded so that numerical measures of the rats' performance could be obtained later.

Navigational pretraining. The purpose of pretraining was to teach rats that a buried food reward could be obtained by digging into the sawdust. At the beginning of pretraining, an uncovered 3$\mathrm{cm}$-diameter dish containing seven Coco Puffs was put onto the surface of the sawdust, next to a $13-\mathrm{cm}-\mathrm{high}$ dowel. The rat explored the initially unfamiliar cylinder, eventually got to the food dish, and ate. On subsequent trials, the food dish + dowel combination was moved randomly within the cylinder. On each trial, the dish was pushed further into the sawdust. On the trial in which the dish was nearly buried, it was covered with a close-fitting lid and sealed with a piece of Parafilm; sealing the food was done in all subsequent pretraining and in all experimental trials. On each trial, the rat was released at the wall from one of the four cardinal points in the cylinder.

After a week with four trials per day, each rat learned to run quickly to the dowel and to dig deeply at its base to obtain the food reward. Since the location of the food + dowel was moved randomly on each trial, the rat had to use a beacon strategy (go to the dowel) to get the food. If the rat dug at the base of the dowel and obtained food on two successive trials at the end of the week, it was tested on a catch trial in which no food was buried. If the rat ran to the dowel (as was almost always the case), the rat was finished with pretraining.

Preoperative navigational training. Navigational training was designed to teach the rat to go to a fixed, unmarked location to retrieve food under the sawdust. During preoperative navigational training, the goal location for a given rat was the same on every trial. The location for a rat was randomly chosen from a list of 16 
possible locations. The 16 possible locations were at the vertices of two octagons whose centers were at the cylinder center. The vertices of the smaller octagon were halfway out along the appropriate radii; the vertices of the larger octagon were three quarters of the way out along the same radii.

Training to the preoperative goal began by burying the food at the selected location. The rat was put into a small holding box for $2 \mathrm{~min}$. The box was carried to one of the four cardinal points against the cylinder wall, the top was opened, and the rat released. The first navigational trial was the first time there was no dowel to mark the goal location, and there should have been no clues to the food location. On this trial, the rats did very little digging. Not 1 rat (out of 24 used in both navigational experiments) found the hidden food in the 3 -min trial duration allowed in the first navigational experiment or in the 2-min trial duration allowed in the second navigational experiment, indicating that the rats were not using cues emanating from the goal. After the maximum time elapsed, the experimenter entered the controlled cue environment and raised the food vertically to the sawdust surface. The rat walked to the goal location where it was permitted to eat four of the seven buried Coco Puffs. On subsequent baited trials, the rat either found the food within the allotted 2 or $3 \mathrm{~min}$, or the food was raised at the end of the maximum period.

After a trial, the rat was returned to its home cage for $1-2 \mathrm{~h}$ or overnight. It was practical to do three or four of the brief trials for each of 12 rats in a day. Preoperative navigational training was finished after 40 trials run in about 12 days (first navigational experiment) or 30 trials run in about 8 days (second navigational experiment). At the end of this training phase, each rat routinely ran directly to the goal location and successfully obtained food.

During preoperative training and most of the postoperative testing, every third trial was a rewarded "catch" trial. No food was buried in such a trial, but if the rat dug at the goal location, the experimenter quickly entered the controlled-cue curtains and put a food dish on the top of the sawdust at the correct goal location. Catch trials were used to test whether the rat was navigating to the goal or whether it was using information available from the food, the dish, or anything else local to the correct goal. It is an important part of the experimental design that the rat was eventually rewarded at the correct place on every ordinary trial.

Assessment of performance was done by timing how long it took a rat to (1) dig within $5 \mathrm{~cm}$ of the goal and (2) find the buried food. During unrewarded catch trials at the end of experimental phases, we also measured how long the rat spent in each quadrant of the cylinder (Figure 9).

Rats were given from 30 to 60 preoperative training trials. After 30 trials, training was terminated when a rat reached criterion of averaging less than $10 \mathrm{sec}$ to find food in a five-trial block. If the rat didn't reach this criterion by the 60th trial, training was terminated.

Training-lesion interval. The major difference between the two navigational experiments was the interval between preoperative training and the time at which lesions were made. In the first experiment, the lesions or sham lesions were made between 2 and 6 days after the end of training lesions. For convenience, this is called a 1 -week training-lesion interval. In the second navigational experiment, the training-lesion interval was 14 weeks, even though the interval differed by a few days across rats.

Postoperative navigational testing. After surgery (described below), rats were allowed 10 days to recover before additional navigational experience. In both the 1 - and 14-week experiments, 30 trials were done with the original, preoperative goal; again, every third trial was an unbaited catch trial. After this point, the two experiments diverge in design.

In the 1-week navigational experiment, the lesioned rats were incapable of finding the original hidden goal after 30 trials. We therefore gave them an additional 20 trials with the original goal to see whether they would eventually relearn to get the buried food; no unbaited probe trials were done in this phase of the experiment. In addition, hidden-goal trials were alternated with dowel-marked trials to see whether showing the rats the goal location in close temporal proximity to unmarked trials would help them to navigate to the hidden goal.

In the 14-week navigational experiment, an unrewarded hiddengoal trial was done at the end of the 30 postoperative trials at the original goal location. The purpose of this trial was to see how rats distributed their time and digging behavior when a correct dig at the goal location did not terminate an unbaited trial. In the next postoperative phase of the 14-week navigational experiment, a new goal was selected for each rat and transfer training was initiated. Thirty trials were done at the new goal location, followed by another unrewarded hidden-goal trial. Finally, the original goal was used again for 20 trials.

\section{Method for Object Discrimination Experiment}

Apparatus. The apparatus was a gray wooden square, $140 \mathrm{~cm}$ on a side and $100 \mathrm{~cm}$ high. In this case, no intentionally introduced cues were used to distinguish the walls from each other. The square was positioned in the same controlled-cue environment used for the navigational experiments. The floor of the apparatus was covered with $10 \mathrm{~cm}$ of sawdust mixed with ground Coco Puffs. The sawdust and the Coco Puff powder were replaced once per week.

Pretraining. The method was exactly the same as for navigational pretraining; the purpose of pretraining was to teach the rats to dig for food buried at the bottom of a 13-cm-high dowel.

Object discrimination training. This training was designed to teach the rat to dig for food buried under 1 of 10 small objects placed on the sawdust surface. Rats were trained for 1 week to find food buried under a single small object moved from place to place on the surface of the sawdust. After the Ist week, 9 other objects were added. The position of the 10 objects on the sawdust surface was randomized for each subsequent trial, but food was located only under the original object. Examples of the objects are an empty 12-oz. soda can, a small drinking glass, and a plastic hotdog + bun. On each trial, the objects were placed at 10 locations randomly selected from 16 possible locations arranged as a square grid whose sides were $100 \mathrm{~cm}$ long. On the first preoperative training trial. At the end of the maximum searching time of $3 \mathrm{~min}$, the experimenter entered the controlled-cue environment and raised the buried food vertically next to the correct object. On subsequent baited trials, the rat either found the food by itself or, after $3 \mathrm{~min}$ had passed, was shown the buried food by raising it to the surface and allowing the rat to eat. Every third trial was a probe trial with no food buried. If the rat dug at the correct object, the experimenter quickly put the food cup on the sawdust next to the object. If it failed to dig at the correct object after $3 \mathrm{~min}$, the experimenter entered the controlledcue environment and placed the food next to the right object. Thus the rat was rewarded on every pretraining trial.

The rats' performance during training was scored according to the number of incorrect digs. An error was counted if the rat either displaced or dug next to one of the incorrect objects. The number of errors on a trial is the sum of the number of incorrect digs made either before a correct dig or before the maximum time had passed. At the end of 30 trials done over 2 weeks, 1 rat failed to dig correctly on 9 of the last 10 trials and was excluded from the study. All other rats performed almost perfectly at the end of 30 trials and each dug at the correct object on the last preoperative trial.

Training-lesion interval. The interval between preoperative training and surgery for the object discrimination experiment was the same as for the first navigational experiment- 1 week. Prior to surgery, 6 rats were scheduled to have lesions and 5 to have sham lesions. One of the lesioned rats died after surgery. For this reason, 1 rat scheduled to have sham lesions was instead given actual le- 
Table 1

Injection Coordinates

\begin{tabular}{cccc}
\hline & AP & ML & DV \\
\hline 1 & -2.5 & 1.4 & -3.7 \\
2 & -2.5 & 2.1 & -3.7 \\
3 & -3.3 & 1.5 & -3.7 \\
4 & -3.3 & 2.5 & -3.7 \\
5 & -4.1 & 3.2 & -4.2 \\
6 & -4.1 & 4.0 & -4.2 \\
7 & -4.8 & 3.2 & -4.1 \\
8 & -5.3 & 4.8 & -6.0 \\
9 & -5.8 & 5.0 & -5.0 \\
10 & -5.8 & 5.0 & -7.3 \\
\hline
\end{tabular}

Note-All measurements are in millimeters, from bregma.
Sections through the same places in a lesioned brain (Figures $\mathrm{IC}$ and D) reveal the almost complete absence of the hippocampus and dentate gyrus. Only a small remnant of the ventral hippocampus $(V)$ is visible. Since the section from the lesioned animal is typical, there is little doubt that the damage to the hippocampus and dentate gyrus is massive.

Excitotoxic lesions of the hippocampal formation can induce seizures that damage the entorhinal cortex (Jarrard \& Meldrum, 1993). The postsurgical treatment with diazepam was aimed at minimizing this type of seizure-induced damage. In examining retrohippocampal regions of the lesioned rats, we found no detectable damage in the entorhinal cortex or other areas removed from the injection sites. sions to keep the lesioned group size at 6 . The number of sham lesioned animals was therefore 4

Postoperative testing for object discrimination. After 7 10 days for recovery, rats were tested for their ability to find food buried under the correct object. The first three trials were done on a single day under extinction conditions; in these trials, no food was buried and no food was given near the object after a correct dig. For the next 2 days, the preoperative reward protocol was resumed for a total of 10 trials.

\section{Surgical Method}

Rats were anesthetized with pentobarbital $(35 \mathrm{mg} / \mathrm{kg}$ ), placed in a Kopf stereotaxic instrument, and given either biochemical lesions or sham lesions. The aim of the biochemical lesions was to destroy as much of the hippocampus (Ammon's horn proper) and the dentate gyrus as possible, along the entire septal-temporal extent of the hippocampus. This was done by injecting $0.1 \mu \mathrm{l}$ of a cytotoxic solution $(0.8 \mu \mathrm{g}$ of colchicine and $0.04 \mu \mathrm{g}$ of kainic acid) through glass pipettes at each of 10 sites on each side of the brain (Table 1). Fluid was injected using calibrated pressure pulses (Picospritzer II General Valve Corp., Fairfield, NJ). Surgery for sham lesions was identical to surgery for lesions, except that the saline contained neither toxin and no solution was ejected from the pipette.

During the first $6 \mathrm{~h}$ after surgery, rats were given $1.5-2.0 \mathrm{mg}$ of diazepam, in five injections, to prevent seizures and to help restrict damage to the injection sites (Sutherland et al., 1983). No seizures were seen during recovery from anesthesia. About 10 days were allowed for recovery from surgery, during which time each rat was handled once per day. The hippocampally lesioned rats (but not the sham lesioned rats) were jumpy for $2-4$ days after surgery, but after 10 days were calm and easily managed.

Analysis of lesions. At the end of the experiments, all rats with hippocampal lesions and several sham lesion controls were killed with an overdose of pentobarbital and perfused transcardially with physiological saline followed by $10 \%$ formaldehyde in saline. The brains were removed and 50-micron frozen coronal sections were taken along the entire length of the hippocampus and stained with cresyl violet. Every fifth section was converted into a digital image using a microscope equipped with a television camera and a video frame grabber, and the area of the hippocampus proper and dentate gyrus was outlined. Volume measurements calculated from these areas are summarized in Table 2 . Selected sections were also photographed to provide examples of the extent of the lesions caused by the toxic solution injections.

The appearance of a brain subjected to sham lesions is shown in Figures $1 \mathrm{~A}$ and $\mathrm{B}$. The appearance of the dorsal hippocampus and dentate gyrus (D) is normal in this coronal section at $5.2(A)$ and 3.0 (B) $\mathrm{mm}$ anterior to earbar zero. Section $B$ also cuts through the ventral hippocampus (V) at the bottom. These sections correspond fairly well to plates 33 and 41 in the Paxinos atlas (Paxinos, 1986).

\section{RESULTS}

\section{Experiments 1 and 2: \\ Effects of Massive Hippocampal \\ Lesions on Recently Learned Tasks}

In these experiments, rats were trained for 2 weeks on one of two tasks. They were then subjected to either sham lesions or combined biochemical lesions of Ammon's Horn and the dentate gyrus 2-4 days after the completion of training. As documented in the Method section, the lesions destroyed more than $90 \%$ of the hippocampus and dentate gyrus, but largely spared entorhinal cortex. When the ability to retain or relearn the original task was tested beginning 1 week after surgery, the massive lesions devastated performance on a navigational task but had little effect on an object recognition task.

\section{Experiment 1: Lesions Made Shortly After Training Severely Impair the Ability to Navigate to a Familiar Hidden Goal}

The rats in the hippocampally lesioned and sham lesioned groups learned to navigate to the hidden goal at the same rates during preoperative navigational training. Figure 2 plots the first 40 training trials prior to the time when any rat had reached criterion and was removed from training. For Trials $20-40$, the first dig for each rat was correct and food was recovered on all baited trials.

Ten of 12 rats reached criterion (averaging less than $10 \mathrm{sec}$ for a five-trial block) by the 60th trial. One reached criterion in Block 36-40, 1 in Block 41 - 45, 5 in Block 4650 , and 3 in Block 51-55. The 2 rats that never reached criterion did find food on each of the final 40 training trials, but their behavior was contaminated by occasional trials, about one in three, with very long latency. These 2

Table 2

Histology Results: Mean Percent Control and Standard Deviations

\begin{tabular}{|c|c|c|c|c|c|c|}
\hline \multirow[b]{3}{*}{ Brain Area } & \multicolumn{6}{|c|}{ Experiment } \\
\hline & \multicolumn{2}{|c|}{1} & \multicolumn{2}{|c|}{2} & \multicolumn{2}{|c|}{3} \\
\hline & $M$ & $S D$ & $M$ & $S D$ & $M$ & $S D$ \\
\hline Hippocampus* & 5.5 & 1.6 & 4.4 & 2.7 & 4.4 & 2.0 \\
\hline Dentate gyrus ${ }^{\dagger}$ & 7.9 & 3.2 & 6.0 & 3.1 & 6.9 & 3.9 \\
\hline
\end{tabular}

${ }^{*}$ Control brain volume (one side) $=26.8 \mathrm{~mm}^{3}$. ${ }^{\dagger}$ control brain volume (one side) $=11.4 \mathrm{~mm}^{3}$. 


\section{Sham}
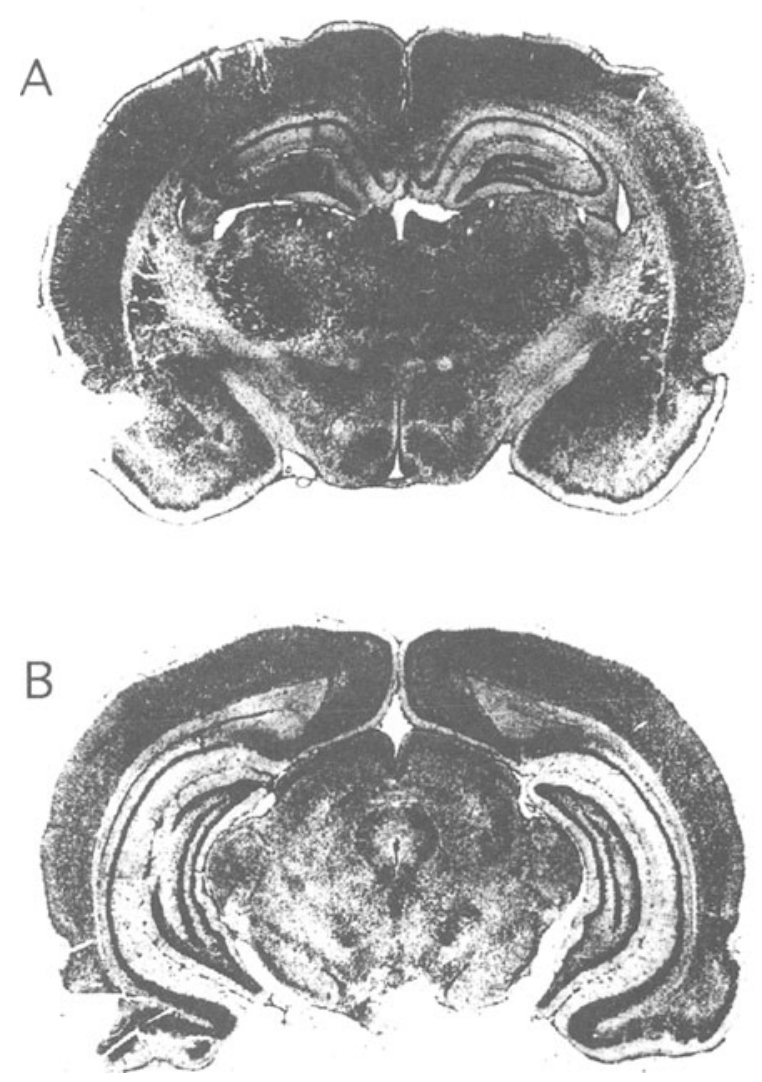

\section{Hippocampal Lesion}
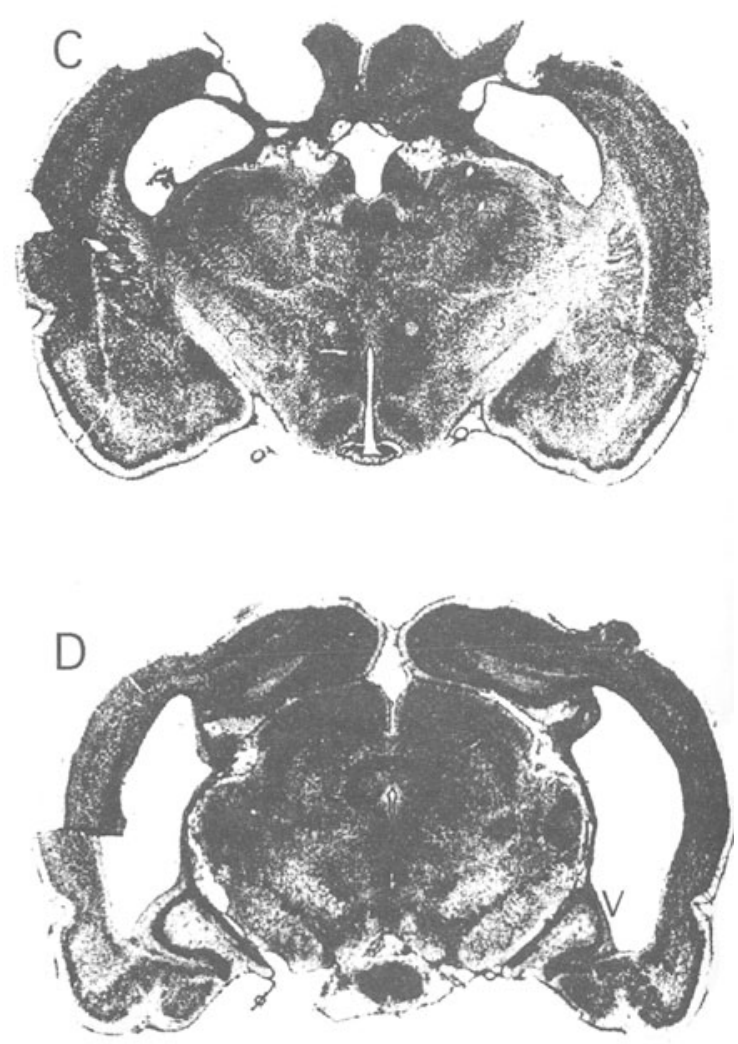

Figure 1. Photographs of coronal sections at 5.2 (A) and 3.0 (B) $\mathrm{mm}$ anterior to earbar zero from the brain of a sham lesioned rat. (A) shows the dorsal hippocampus and dentate gyrus (D). The section in (B) also cuts through the ventral hippocampus (V) at the bottom. These sections correspond fairly well with plates 33 and 41 in the Paxinos and Watson atlas (Paxinos, 1986). Sections taken at corresponding levels in a lesioned brain ( $C$ and $D)$ reveal the almost complete absence of the hippocampus and dentate gyrus. Only a small remnant of the ventral hippocampus is visible $(V)$. The sections from the lesioned animal are typical.

rats were eliminated from the study, leaving 5 animals in each surgical group. The right side of Figure 2 plots the final 10 training trials for the 10 rats that reached criterion. Over this interval the two groups are equivalent $[F(1)=0.242, p>.1]$, and group means appear to be approaching an asymptote under $10 \mathrm{sec}$ to dig in the correct location.

Behavior on trials in which no food was buried ("probe trials") was indistinguishable from baited trials, as expected if the rats navigate to the goal rather than going toward odors available from the food or by using tactile cues as they walk over the food cup at the goal.

Surgery was done over a 5-day interval starting 1 day after the end of training. Beginning 1 week after surgery, rats were tested for their ability navigate to the original goal location. The initial postoperative performance for sham lesioned rats was poorer than at the end of training (Figure 3). We did not investigate whether this was due to the time interval alone, to the effects of anesthesia, or to the combined effects of anesthesia plus multiple penetrations by the micropipette. Nevertheless the sham lesioned rats relearned to find the buried food, and their performance was slightly better after 30 postsurgical trials than preoperatively.

In contrast to the sham lesioned group, the lesioned group could not relearn to go to the original location (Figure 3). On 16 of the first 30 postoperative trials, not even 1 lesioned rat dug within $5 \mathrm{~cm}$ of the goal within $180 \mathrm{sec}$. In the few cases when the distance criterion was satisfied, the dig was shallow and lesioned rats found the food only two times in 150 trials. Note that even though the digging was shallow and not concentrated near the goal, the fact that the lesioned rats dug at all implies they expected food; naive rats do not spend time digging otherwise.

Lesioned rats might have been unable to find the buried food because they could not solve the navigational problem or because they were less motivated to find the food, because they ran more poorly, and so on. We there- 


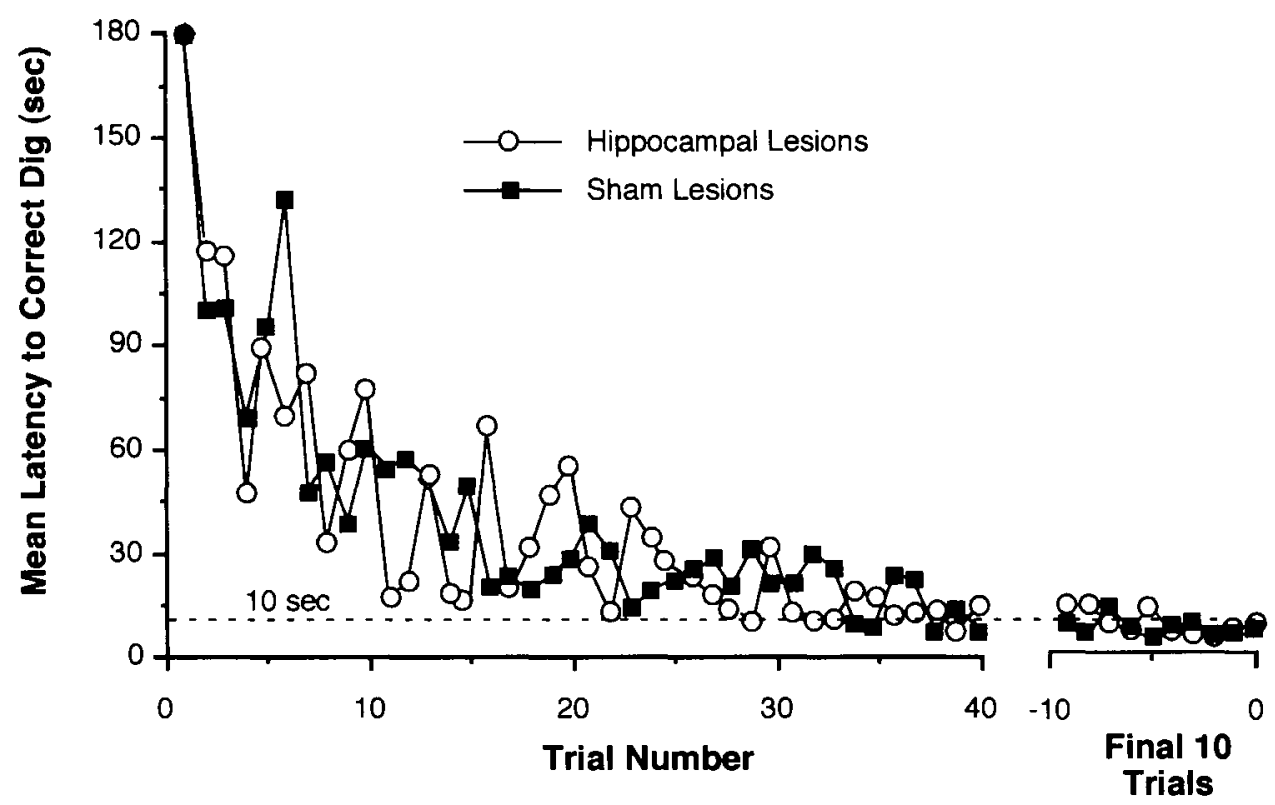

Figure 2. Preoperative training for rats in Experiment 1 . The mean latency to dig within $5 \mathrm{~cm}$ of the goal is shown for rats scheduled to have hippocampal lesions (open circles, $n=5$ ) or sham lesions (closed circles, $n=5$ ). The 2 rats that did not reach criterion are excluded from this plot. Digging latencies decreased with training and, for the final 10 trials, reached an asymptote of about 15 sec. The right side of the figure plots the final 10 trials, prior to reaching criterion.

fore asked whether lesioned rats could relearn to go to the food location when it was marked by a vertical dowel. This is analogous to the visible platform variant of the Morris swimming task (Morris, 1984). With the dowel present, the performance of lesioned rats was excellent (Figure 4). The very short latency to dig near the correct location on the first few dowel-cued trials suggests that the lesioned rats remembered from pretraining that the dowel signals food location and is in agreement with the object discrimination task results. The fine performance during dowel-cued trials shows that the rats were motivated, that they knew food was available, and that there were no motor impairments.

By alternating marked and unmarked trials, we also asked whether successful performance on cued trials could help improve performance on interposed unmarked trials. As is evident from Figure 4, the lesioned rats still could not find the food location when it was unmarked, even though they went directly to the same location on cued trials.

\section{Experiment 2: Lesions Made}

\section{Week After Training Do Not Disrupt an Object Discrimination Task}

During the 30 trials of preoperative training in the object discrimination task, rats learned to dig near the baited object. They behaved identically on normal trials and on catch trials, indicating that they were digging near a selected object and not directly detecting the buried food (Figure 5).
After surgery, rats were given three trials with no buried food. All rats showed retention of object discrimination ability during these trials (Figure 5). In fact, on the first postoperative trial, every lesioned and every sham lesioned rat chose the correct object, showing perfect retention. Perhaps due to extinction, performance deteriorated slightly on the next two postoperative probe trials. Nevertheless, the overall performance level was much better than chance for both groups. Thus, rats in both groups retained the ability to locate food buried under a specific object; relearning was unnecessary. The lack of a deficit in the lesioned animals is further shown by their excellent performance in rewarded trials; during the last five postoperative trials for the lesioned rats, the correct object was the first choice on $26 / 30$ trials. Thus, retention of an object discrimination is possible after massive lesions of the hippocampus plus dentate gyrus. The lack of an effect of hippocampal lesions on retention of an object discrimination is largely consistent with findings from monkey and rat studies (Mumby, Wood, \& Pinel, 1992; Vnek \& Rothblatt, 1996; Wible, Shiber, \& Olton, 1992).

\section{Experiment 3: Effects of Lesions Made Many Weeks After Training}

In the third experiment, two groups of 6 rats were trained in the same buried food task as that used in the first experiment. As in the first experiment, rats were eventually rewarded in each trial. Both groups learned the task rapidly-more rapidly than the rats of Experiment 1 . Eight of the rats reached training criterion in Block 25-30, 


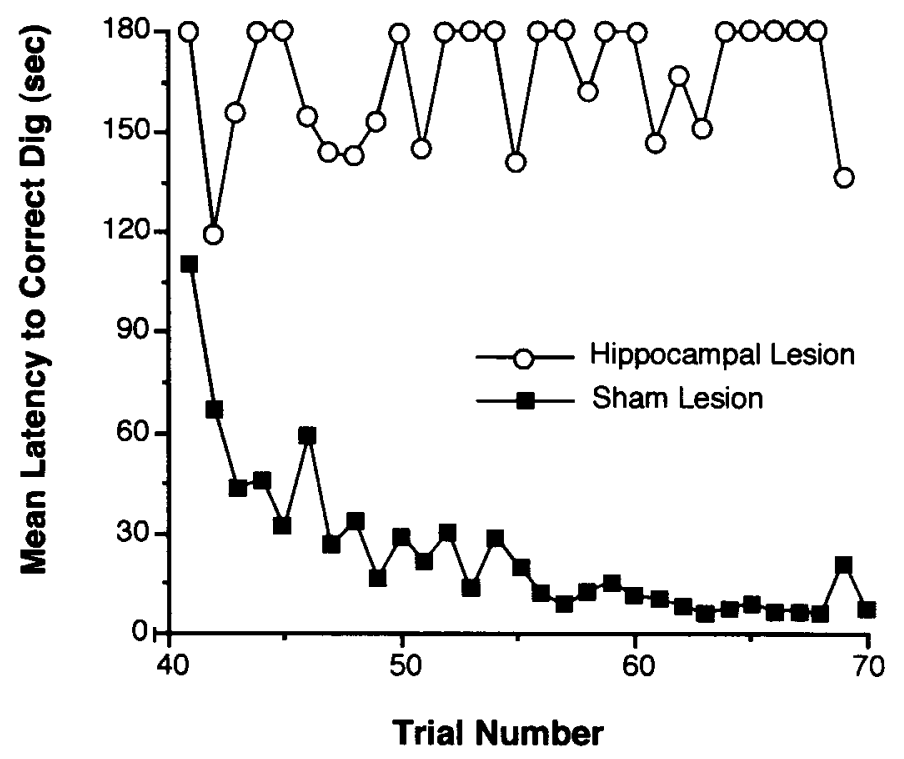

Figure 3. Postoperative performance for rats in Experiment 1 (1-week training-lesion interval). Both groups of rats had surgery within 3 days after the completion of initial training (Figure 2). Rats in the sham lesioned group rapidly relearned the goal location, whereas rats with hippocampal lesions showed no postoperative reacquisition. In trials where the mean latency was $180 \mathrm{sec}$, none of the 6 rats dug within $5 \mathrm{~cm}$ of the goal. Rats in the hippocampal lesioned group occasionally dug near the goal, but these appeared to be random guessing.

and the remaining rats reached criterion in Block 31-35. The rapid training is apparent in Figure 6, which plots the first 30 trials, when all rats were in training. There is an apparent asymptote by Preoperative Trial 20, and over the final 10 plotted trials $(21-30)$, every rat found food on the first dig with an average latency of $6.7 \mathrm{sec}$. A twoway repeated measures analysis of variance (ANOVA) on the final 10 trials revealed that performance was indistinguishable for the rats assigned to the two surgical procedures $[F(2)=2.90, p>.1]$; it was also the same on baited and probe trials.

\section{Experiment 3A: Rats With a 14-Week Training-Lesion Interval Can Find the Hidden Goal if It Is at Its Original Location}

After a 14-week training-lesion interval, rats were subjected either to hippocampal lesions $(n=6)$ or sham lesions $(n=6)$ followed by a 1 -week recovery period. The rats were then tested for the ability to navigate to the original hidden food location. As in Experiment 1, initial performance was not as good as at the end of training (Figure 7A). The long initial latencies reflect the performance by each rat in each group; for example, no rat made its first dig at the correct place in the first postoperative trial.

With additional training, rats with sham lesions showed marked improvement and performed at the final preop- erative level after about $18-20$ postoperative trials. Lesioned rats also relearned the task, but more slowly than the sham lesioned group. An ANOVA run on the final 10 trials of the block revealed significant group differences $[F(2)=21.6, p<.01]$. Initially, digging close to the goal location appeared to be the result of frequent shallow digs over much of the apparatus area. With the reinforcement given at the end of each trial, however, digging began to be confined to the region near the goal. After about 24 postoperative trials, each lesioned rat almost always dug first at the correct goal location and dug deeply enough to retrieve the buried food. As during preoperative training, every third trial was a probe trial in which no food was buried. None of the rats behaved differently on probe trials, showing that the rats were not finding the food by using cues local to the goal.

Performance near the end of postoperative retraining (on Trial 25) is shown for individual lesioned and sham lesioned rats in Figure 8A. The line in each panel shows the path the rat took from the beginning of the trial to when it found food. Each rat took a fairly direct path to the buried food location, and in fact the average path length for the lesioned rats happened to be somewhat shorter than that for the sham lesioned rats on this trial.

A further indication that the lesioned rats knew the original unmarked goal location at the end of postoperative retraining is their tendency to stay in the goal quadrant during an unrewarded probe trial after the 30th re- 


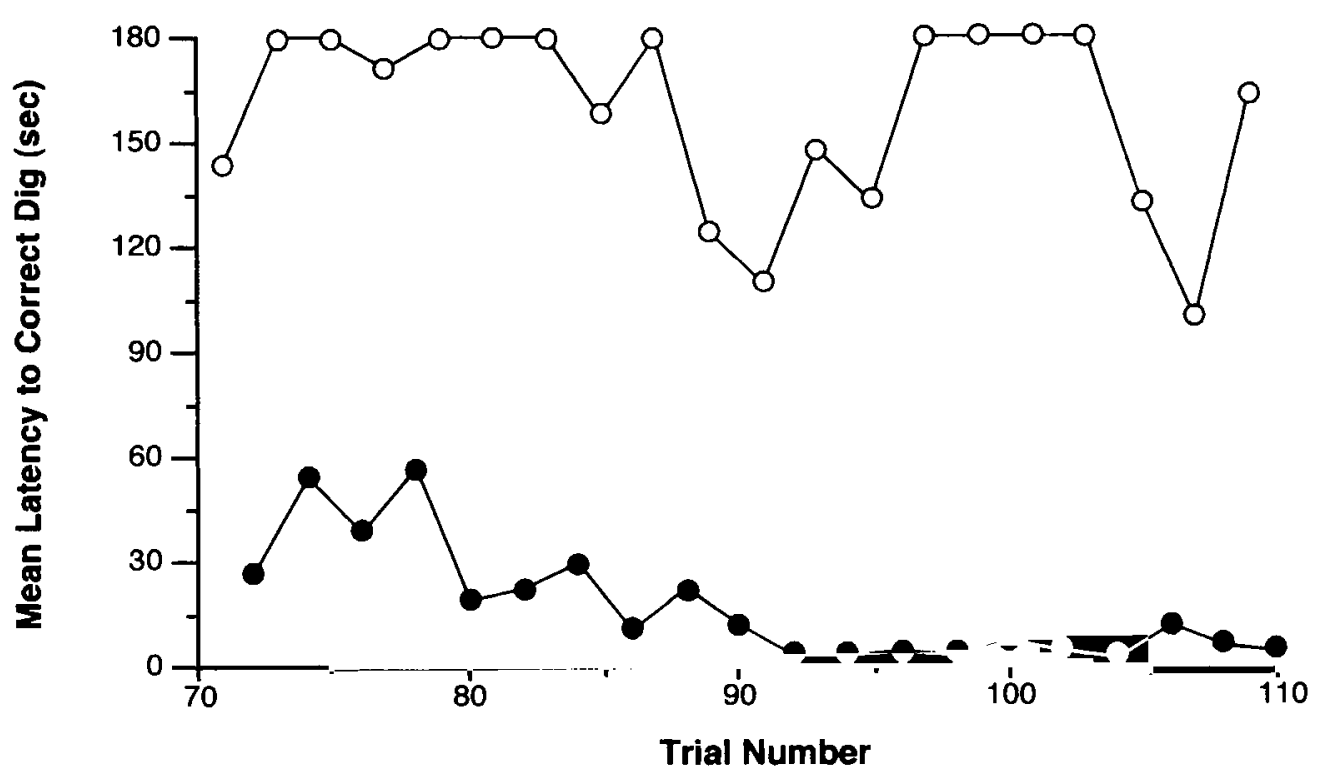

Mean with No Dowel

Figure 4. Postoperative performance in Experiment 1. Alternating marked and unmarked trials does not help rats with a 1-week training-lesion interval to relearn to get to the original goal. Training was extended for these rats with unmarked trials alternated with trials in which a wooden dowel marked the location of the buried food. Rats with hippocampal lesions immediately made effective use of the marker and found buried food on all dowel trials. By the end of this segment of training, rats were finding the dowel-marked goal in under 10 sec. Dowel training, however, had no effect on unmarked trials: The rats with a 1-week training-lesion interval remained completely unable to find the buried food when no visible marker was present.

training trial. The lesioned rats did not simply go to the goal, dig once, and then roam randomly. Instead, as shown in Figure 9, the lesioned as well as the sham lesioned rats confined their search mainly to the region near the correct goal; each lesioned rat acted as if it expected to find food at the goal.

The main conclusion from Experiment $3 \mathrm{~A}$ is seen by comparing the performance of lesioned animals with 1 and 14-week training-lesion intervals. First, we want to compare preoperative training for the rats in the two experiments. For reasons we cannot explain, rats in Experiment 1 learned the task more slowly than rats in Experiment 3. Nevertheless, by the end of preoperative training, the two groups were finding the buried food equally well. Figure 10 plots the mean latency to find buried food for the final 10 trials of preoperative training for the four groups of rats (sham and lesion, Experiment 1; sham and lesion, Experiment 3). The rats trained in Experiment 1 (sham plus lesion) were indistinguishable from the rats of Experiment 3 (lesion plus sham) [repeated measures ANOVA, $F(1)=2.98, p>.1]$. Although the rats in the two experiments were not perfectly matched in terms of the time necessary to learn the task, by the end of preoperative training, they exhibited equivalent performance.

Performance was comparable by the end of preoperative training, but dramatic differences emerged postop- eratively. Lesions made 1 week after training permanently devastated performance, whereas lesions made 14 weeks after training only slowed relearning (Figures 2 and $7 \mathrm{~A}$ ). All rats in the latter group relearned to navigate effectively, whereas none of the rats in the former group showed any evidence of spared navigational capacity. We conclude that the hippocampus is required for navigation if only a brief interval is interposed between training and testing but is not necessary for adequate performance if sufficient time elapses between training and testing. In short, the difference in performance between the short and long training-lesion intervals suggests that retrograde amnesia is graded.

\section{Experiment 3B: Postoperative Performance With the Hidden Goal at a New Location}

Since rats with a 14-week training-lesion interval could find the original goal, we asked whether they could learn to find a new goal in the same environment. Accordingly, each rat in each group was assigned a different goal location randomly selected from 15 sites that remained after the original site was precluded. Transfer training was done for 30 trials over a 2-week interval.

As shown in Figure 7B, sham lesioned rats rapidly transferred, digging at the new goal on every trial after the first and successfully retrieving the food on the first dig. The lesioned rats behaved entirely differently. At the 


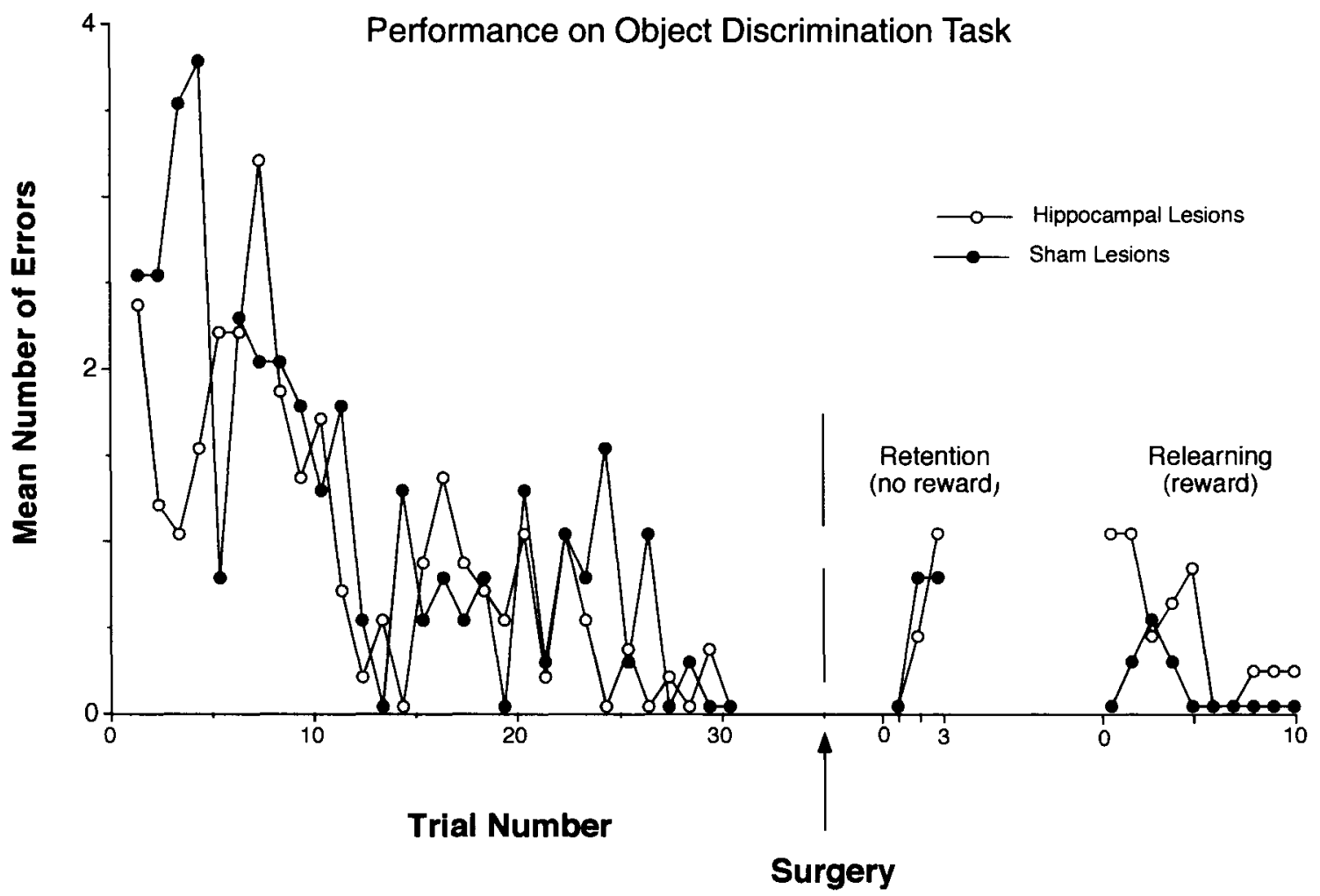

\begin{abstract}
Figure 5. Massive hippocampal lesions made 1 week after training did not affect performance in the object discrimination task. Open circles depict performance of rats in the hippocampal lesion group $(n=6)$; closed circles depict performance of rats in the sham lesioned group $(n=4)$. The left side of the figure shows performance prior to surgery while both groups of rats gradually learned to dig at the correct object. The aggregate performance starts better than chance (five errors) due to pretraining with the goal object. By the final five-trial segment of training, rats dug first at the correct object $92 \%$ of the time. Following surgery and recovery, three retention trials were run, with no reward available until after object selection in the third trial. Performance of both groups of rats closely matched preoperative levels, and, in fact, was perfect for all animals on the first postoperative trial. Since these trials were run under extinction conditions, it is not surprising that performance deteriorated somewhat by the third trial. Rats were given a final block of 10 trials with the correct object selection rewarded. Performance of both groups of rats rapidly returned to presurgical levels.
\end{abstract}

outset of transfer training, they tended to dig first at the original goal and would often make additional digs there. There seemed to be nothing systematic about digging near the new goal location. The digs were shallow and were preceded and followed by equally shallow digs virtually anywhere in the apparatus. Interestingly, the amount of digging decreased with repeated trials using the new goal location; the digging behavior seemed to extinguish. There was no evidence that the rats learned the new location by the end of the transfer trials; in the final 10 transfer trials, lesioned rats found the food only three times $(5 \%$ of 60 attempts).

The great difference in performance between lesioned and sham lesioned rats with the new goal location is evident from Figure 8B, which shows paths for the 25th posttransfer trial. The paths taken by the sham lesioned rats were direct and appeared to be the same as paths taken to the original goal (Figure 8A). In contrast, the lesioned rats wandered all around the apparatus and often passed over the goal location without digging at all or making digs too shallow to possibly yield food; there is no indication they knew about location of the goal. The tendency of the lesioned rats to walk right through the goal location was rendered visible by reducing the thickness of the drawn line the first time the goal location was traversed.

We conclude that rats with hippocampal damage cannot learn to navigate to a novel goal location even in a familiar environment. The failure of the rats to learn the new goal location is a form of anterograde amnesia. It appears that the lesioned rats showed both the graded retrograde amnesia and the anterograde amnesia associated with the human amnestic syndrome.

\section{Experiment 3C: Retraining to the Original Goal}

The ability of lesioned rats to relearn the original goal and their inability to learn a new goal could have been caused by a slow deterioration in their navigational capacity. We tested this by switching back to the original goal for 2 sham lesioned rats and 4 lesioned rats; the other rats were not tested due to time constraints. As 


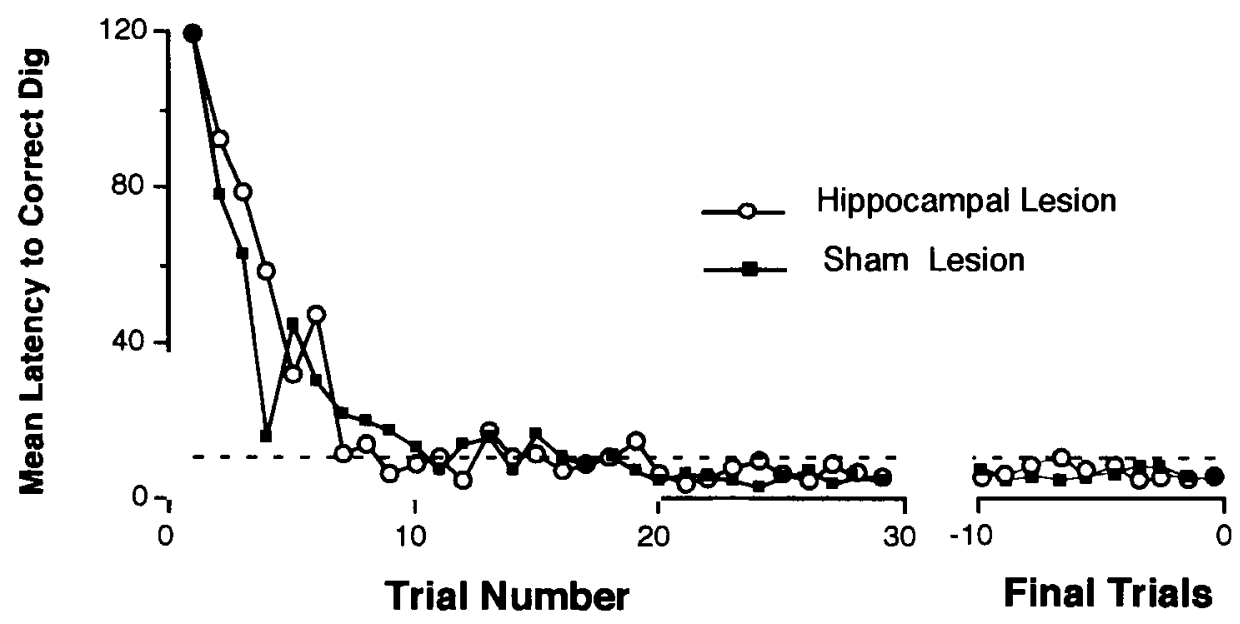

Figure 6. Preoperative training for rats in Experiment 3. Ten of 12 rats reached criterion by the 30th trial. Rats in both groups rapidly learned to dig at the hidden goal on baited trials and unbaited probe trials (Trials 3, 6, 9, etc.). The mean latency for a correct dig over the last 10 trials was 6.7 sec. The right side of the figure plots the final 10 trials prior to reaching criterion.

shown in Figure $7 C$, both sham lesioned animals relearned the original goal very rapidly. The relearning for the lesioned rats was almost as quick. The ability of the lesioned animals to reacquire the original goal is evidence against the idea that the poor performance of the lesioned animals with the new goal was due to some degenerative process.

\section{DISCUSSION}

The Discussion section is divided into three parts. We first provide a narrow view of our results, treating them in terms of the theories expressed by Squire and Alvarez (1995) and by Nadel and Moscovitch (1997) but not comparing them to results from similar experimental work. In the second, we contrast the buried food task to the Morris swimming task. We then consider how our results fit with those from other experiments.

\section{Neocortical Memories Consolidated From Hippocampal Memories Are Not Fully Competent}

We have reinvestigated the retrograde effects of massive lesions of the rat hippocampus on performance in a hidden-goal task similar to the Morris swimming task and on performance in an object recognition task. In both tasks, rats learned to find food buried under a layer of sawdust. In the hidden-goal task, the goal position was fixed in the environment and could be located only by using sensory information not local to the goal. In the object recognition task, the goal was moved around in the environment and could be located only by recognizing and going to one of the 10 objects that was always put above the buried food. The motor activity necessary for successful performance was the same; in both tasks the rat had to walk to the goal location and dig there. Thus, the problems differed in terms of the information and computations necessary to find the goal but not in the actions needed to obtain the food.

Normal rats readily solved both the hidden-goal task and the object recognition task and retained the ability to solve each problem after sham lesions. Performance in the hidden-goal task decreased after a training-operation interval of either 1 or 14 weeks, but sham lesioned rats easily relearned to go to the hidden goal; why performance decreased during the training-operation interval is considered later. Performance in the object discrimination task was unaffected by sham lesions made 1 week after training; the correct object was selected with the same efficiency before and after surgery.

Massive hippocampal lesions made after training had very different effects on subsequent performance depending on both the task and the training-lesion interval. In contrast to sham lesioned animals, rats given massive hippocampal lesions 1 week after training in the hiddengoal task could not relearn the location of the buried food. The severity of the deficit is underscored by the inability of these rats to perform properly even when unmarked trials were alternated with marked-goal trials with the goal at the same place.

Other rats with equivalent lesions had no difficulty with the object recognition task after a 1 week training-lesion interval; their performance was indistinguishable from that of sham lesioned animals. The perfect retention scores in the first postoperative trial argue strongly that this task is nonhippocampal. Thus in agreement with a great deal of previous work, the effects of hippocampal lesions are confined to certain memory tasks.

The most interesting results were seen in the hiddengoal task after a lesion-training interval of 14 weeks. These animals relearned to go to the original hiddengoal location slightly more slowly than rats with sham 

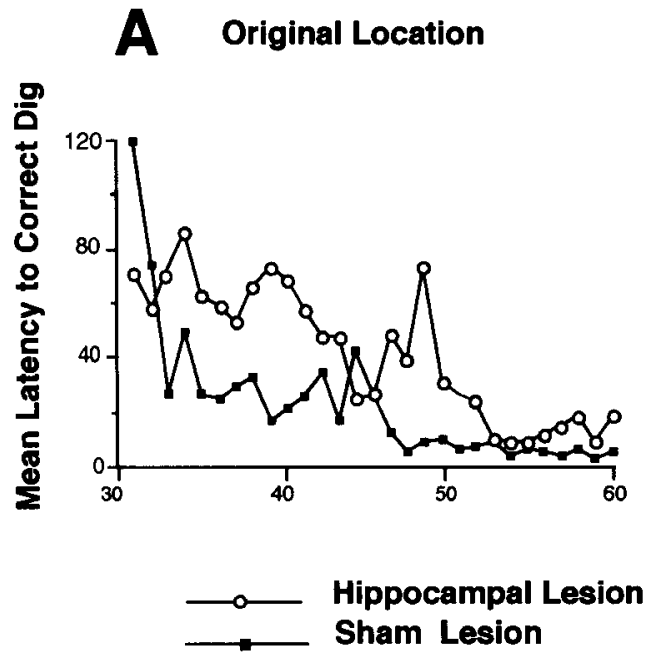

B New Location

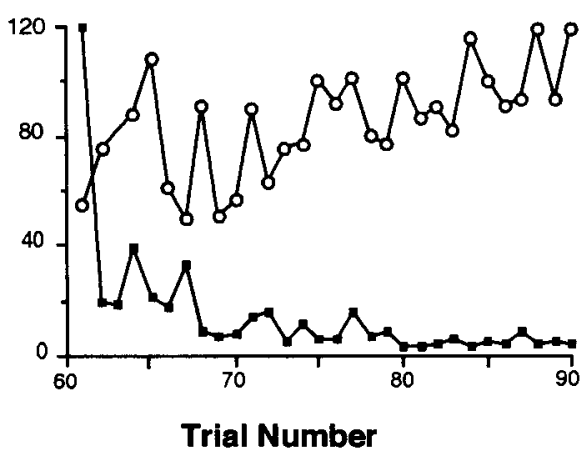

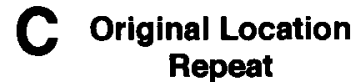

Repeat

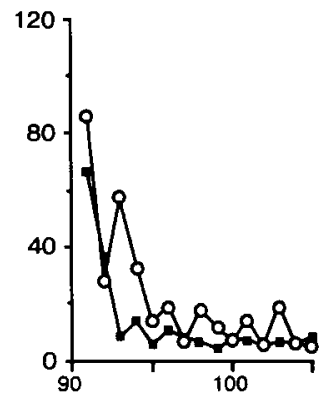

Figure 7. Latency to a correct dig for rats after surgery in Experiment 3 with a 14-week training-lesion interval. (A) Postoperative training to the original goal location. Sham lesioned rats (filled squares) rapidly relearned to dig at the original goal, and by the final block of 10 trials were performing at presurgical levels. Rats with hippocampal lesions had longer latencies to a correct dig than sham lesioned rats, but they consistently found the food. By the final 10 trials, rats with hippocampal lesions dug in the correct location with latencies under $20 \mathrm{sec}$ and found food on all baited trials. Latencies on unbaited trials (Trials $21,23,26$, etc.) were indistinguishable from those on other trials. (B) Postoperative training to a new goal location. Rats with sham lesions (filled boxes) rapidly learned to go to a novel location; rats with hippocampal lesions (open circles) could not learn to find buried food in the new location. Sham lesioned rats learned the novel location about as rapidly as they relearned to find food in the original goal location. Rats with hippocampal lesions performed poorly; their dig attempts were widely scattered and were rarely in the new goal location. Even at the end of this 30-trial block, rats with hippocampal lesions rarely dug in the correct location within the 2 -min time limit, and even more rarely dug deep enough at any location to find buried food. (C) A second period of postoperative training to the original goal location. Rats in both groups rapidly relearned to get to the original goal location. Over the final 10 trials of training, rats in both groups found food on every trial, with mean latencies less than 15 sec.

lesions, but at the end of retraining to the original goal, their performance was as accurate as that of sham lesioned rats. Thus, increasing the training-lesion interval from 1 to 14 weeks produced a dramatic improvement in the ability to get to a hidden goal. This gradient of retrograde amnesia is precisely the sort of result that led to the formulation of consolidation theory. This result implies that the hippocampus is essential for memory storage shortly after learning but that it becomes unnecessary if enough time passes between training and insult. It also implies that the memory for the task is now stored at a nonhippocampal site, often thought to be neocortical.

Since a 14-week training-lesion interval spared the ability to go to the original goal, we analyzed performance after the hidden goal was moved. Rats with sham lesions readily learned to find buried food at the new unmarked goal location. In contrast, the rats with a 14-week training-lesion interval could not learn to get to the new goal. These rats virtually never found the food and began to give up digging so that their latency scores got worse and eventually reached the time limit of $3 \mathrm{~min}$. Nevertheless, when the goal was switched back to the original location, the performance of the 14-week training-lesion rats improved as quickly as that of the sham lesioned an- imals, showing that the failure at the new goal location was not due to overall deterioration.

How can this contrast between performance at the original and relocated goals be explained? One possibility was raised in an interesting paper by Angeli, Murray, and Mishkin (1993), who demonstrated that monkeys with massive hippocampal lesions could solve a variant of the delayed non-match-to-sample task if it was necessary to remember one place, but they could not solve a more complicated variant in which two places had to be remembered. Perhaps we are looking at a similar sort of impairment in which damaged rats can learn at most one hidden goal site. Clearly the task for monkeys is much more complicated, but it is important to test 14-week traininglesion rats for the ability to learn a new goal location if it is presented after the training-lesion interval and before retraining to the original goal. If they can learn the new goal and then cannot relearn the original goal, it would be proper to think of the deficit in terms of reduced memory capacity. This would be a very interesting outcome-it would combine a limited sparing of anterograde memory function with retrograde amnesia and would be hard to reconcile with any current theory. Pending experimental testing of this issue, we assume that performance 


\section{Original Location}

Hippocampal Lesion Rats

Sham Lesion

Rats
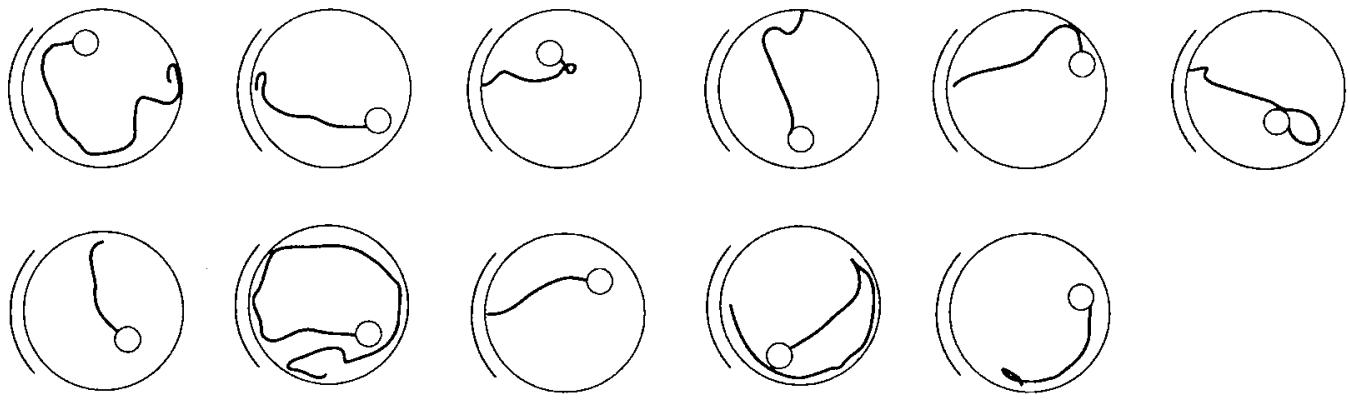

\section{New Location}

Hippocampal Lesion Rats
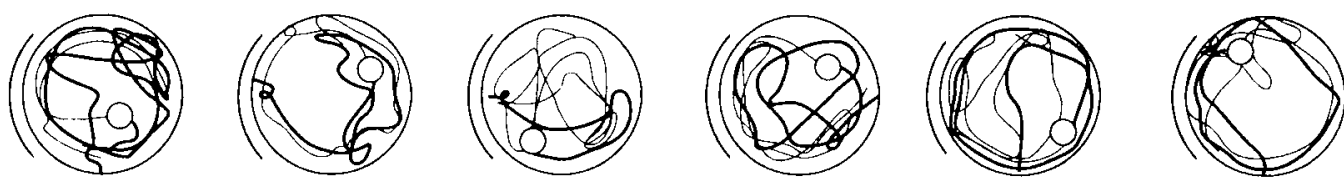

Sham Lesion
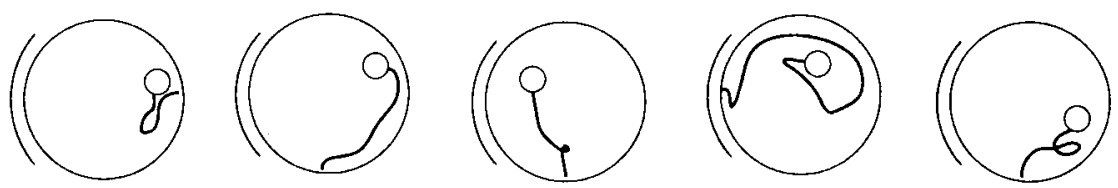

Figure 8. Paths from start to goal for lesioned and sham lesioned rats with a 14-week training-lesion interval. (For 1 rat in the sham group, the videotape was damaged, and no tracings were made.) The top two rows are from the 25th postoperative original location block (Experiment 3A); the bottom two rows are from the 25th trial in the new location block (Experiment 3B). In each panel the small circle shows the goal location. The dark lines show the path prior to arriving at the goal location. If the rat found the buried food, the trace ends at the goal circle, but if the rat continued past the goal, the thick line is replaced with a thin line. During original location training, all rats in both groups took short paths to the goal and found buried food. On the new location trial, the pattern of performance for sham lesioned rats is virtually identical to that of their previous performance: All sham lesioned rats took direct paths to the goal and rapidly found buried food. The pattern of performance for rats with hippocampal lesions was dramatically different. Lesioned rats took meandering paths, and none found the buried food even though they often crossed the goal location.

would be spared for the original location and poor for the new location with either order of testing following the lesions.

A second possibility for explaining the performance difference is that the navigational components of the buried food task are represented in (at least) two different ways, one in the hippocampus and one in the neocortex. In this view, the hippocampus supports a map-like representation of the environment, enabling the rat to learn to get to any goal from any starting place (Muller, Stead, \& Pach, 1996). In contrast, the putative neocortical representation could be based on a vector field (Blum \& Abbott, 1996). Once built, the vectors in the field are arranged so that the rat can arrive at the goal simply by moving in the direction of the vector local to its starting point and continuing to move in the direction of the local vector everywhere along its path. In an open field, the vectors all point directly to the goal. If there is a barrier between the starting point and the goal, the vectors would lead the rat around the barrier. A vector field of this sort therefore permits the rat to get from anywhere in the environment to a fixed goal.'
Blum and Abbott (1996) have shown how a vector field with the required properties can be constructed using the connectivity of the recurrent CA3 network in hippocampus and the properties of long-term potentiation (LTP). In our proposal, the recurrent CA3 network is instead the basis of a "graph" of the environment that is also built using the properties of LTP (Muller, Kubie, \& Saypoff, 1991; Muller, Stead, \& Pach, 1996). This Euclidean graph is a map-like topological representation of the environment that permits the rat to find optimal paths from any starting position to any goal. Thus, we imagine that the hippocampus implements a map and that the vector field representation is neocortical rather than hippocampal. When an intact rat is trained to go to a fixed goal, it constructs and uses a hippocampal map. Without further practice, this hippocampal map can be used to construct a vector field representation in the neocortex. This process takes time and may be explained either as a form of consolidation (Squire \& Alvarez, 1995) or as a consequence of the formation of additional pointers during recalls of the training environment (Nadel \& Moscovitch, 1997). If the hippocampus is removed early after train- 


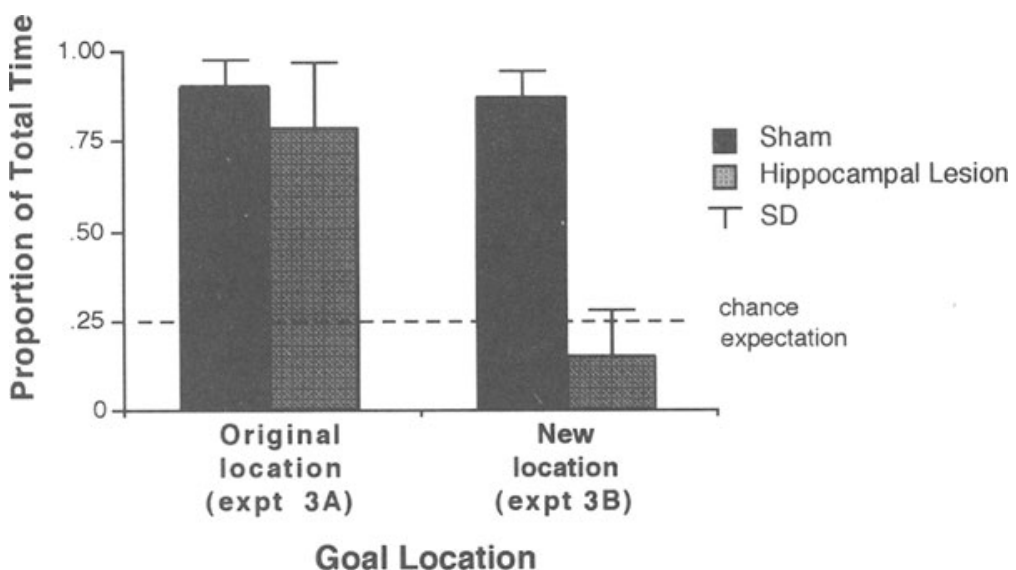

Figure 9. The proportion of the time spent digging in the goal quadrant on probe trials for rats with a 14-week training-lesion interval. At the end of each training block, a 3-minute, unrewarded probe was run for each rat, and the proportion of digging time in the goal quadrant was calculated. Bars depict the mean of these values for sham lesioned rats and rats with hippocampal lesions. On the probe trial run at the end of original goal training, rats in both groups dug almost exclusively in the goal quadrant. In the probe trial run at the end of new location training, rats in the sham lesioned group again dug almost exclusively in the goal quadrant, whereas rats with hippocampal lesions dug indiscriminately, spending less than $25 \%$ of their digging time in the goal quadrant.

ing, the vector field is not fully formed and cannot be used to get to the goal. If enough time elapses before the hippocampus is removed, the full vector field representation permits the rat to relearn the original goal. Because the vector field representation is specific for the original goal, no amount of retraining can cause the vectors to point to a new goal. This is a specific version of the popular notion that the hippocampus permits a kind of adaptability or flexibility in learning (Cohen \& Eichenbaum, 1993; O'Keefe \& Nadel, 1978).

An important corollary to the idea that there are two different ways of representing the navigational components of the buried food task is that the stored information is never a "pure memory" that can be transferred without change from one place to another. Rather, what is stored is specialized for the connectivity, organization, and activity of the brain region in question; at the very least, the information is encoded differently. Thus, the neocortical trace that remains after consolidation and hippocampal damage is not just a copy of the hippocampal trace. Moreover, in the present case it is clear that what comes to be stored in the neocortex is not a fully competent version of the hippocampal trace - after a long training-lesion interval, the rat can solve only a specific case of a more general problem.

This picture is very different from the usual notion of consolidation, in which the memory for an event, a person, an object, or a place is presumed to be transiently held in the hippocampus and then sent in an unchanged form to the neocortex. In the standard view of consolidation, the loss of the hippocampus causes anterograde and graded retrograde amnesia but leaves intact memories acquired a sufficiently long time before hippocampal damage. We suggest, to the contrary, that there will always be ways of interrogating a person with massive hippocampal damage that will reveal deficits. Thus, our position combines elements of the theories of Nadel and Moscovitch (1997) and of Squire and Alvarez (1995); we think that there can be graded retrograde amnesia for hippocampally dependent memory, but that the consolidated information never has the full content after massive hippocampal damage. This conclusion is similar to that of Cho and Kesner (1996).

The possibility that consolidation involves a transformed memory trace rests on the idea that the rodent hippocampus is an "expert system" for navigation. By this we do not necessarily imply that the hippocampus is exclusively devoted to navigation but rather that the full range of navigational computations cannot be performed by other parts of the rat brain. In the same sense, it is our belief that the human hippocampus is also an expert system that is specialized to perform the computations necessary to learn certain kinds of information; it is not merely a transient, passive repository for episodic memory. In short, it is incorrect to think of the hippocampus solely in terms of memory; its unique ability to perform certain kinds of computations means that removal of the hippocampus can leave no memory fully intact.

As an aside, we note that consolidation is often taken to imply that the memory trace is transferred from the hippocampus site to the neocortical so that information about the event is lost from the hippocampus as it is established 


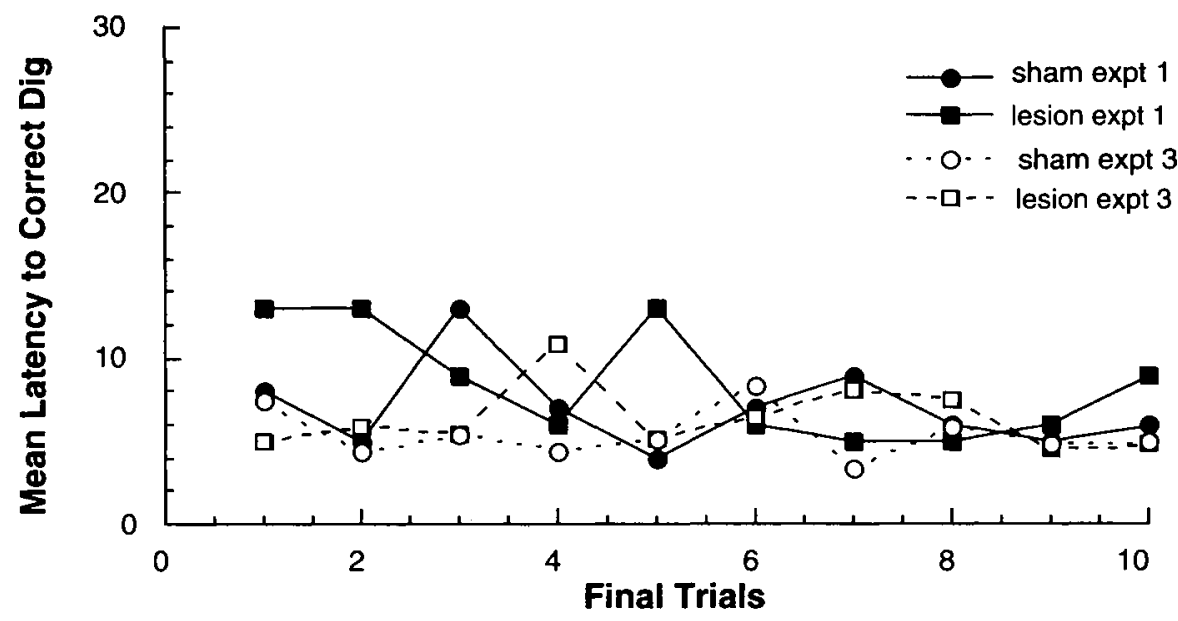

Figure 10. Comparison of final 10 training trials for rats in Experiments 1 and 3. Each rat reached training criterion on the final plotted trial.

in the neocortex. This is in line with the idea that the hippocampus is a temporary memory store. It is important to note, however, that the lesion data do not speak to the issue of whether the hippocampal trace is erased or preserved after the neocortical trace is laid down (Nadel, 1991). It is acceptable to conclude that the hippocampus is no longer needed, but there is no way to tell whether the memory is copied or instead transferred during normal consolidation. In contrast to the ambiguous information provided by lesion data, single-cell work strongly suggests that if consolidation goes on, it does not involve the loss of information from the hippocampus. Thus, recordings from individual place cells (anatomically pyramidal cells) in the rat hippocampus reveal that their firing patterns are stable for weeks or months (Bostock, Muller, \& Kubie, 1991; Muller \& Kubie, 1987; Thompson \& Best, 1990).

\section{The Buried Food Task Compared With the Morris Swimming Task}

The task of finding an unmarked location in which food is buried under a layer of sawdust is similar in many ways to the Morris (1984) swimming task, after which it was designed. In the swimming task, the escape platform is hidden by using milky water and a transparent or white Plexiglas platform. In the buried food task, two precautions are taken to ensure that the goal is unmarked and cannot be reached by going toward signals local to the goal. First, the Coco Puffs used as a food reward are put inside a sealed dish. Second, to mask odors that might nevertheless be detected, many Coco Puffs are crumbled and mixed into the sawdust. With these precautions, rats trained to find buried food at a fixed location virtually never find food buried at other locations, even if they run over the location of the dish (data not shown). Rats that can find the buried food go to the goal location on catch trials even when no food is buried. Also, rats with a l-week training-lesion interval that find and eat buried food at a marked location cannot find food buried at the same lo- cation if it is unmarked, even though all odor and tactile cues from the food and dish are available. Finally, rats with a 14-week training-lesion interval find food only if it is buried at the location used for training; if they were using cues local to the food, their performance would be the same regardless of location. These observations strongly imply that neither sham lesioned nor lesioned rats find food by going toward stimuli that come from the goal location. We also eliminated the possibility that rats use odor trails or other markings from previous trials by thoroughly stirring the sawdust between trials.

In the variant of the dry maze used here, rats were initially trained to go to a fixed location from one of four starting locations, in direct parallel to the most common variant of the swimming task. Later, as is often done in the swimming task, some rats were subjected to transfer training in which they had to learn a new fixed location. As is also the case with the swimming task, many other variations are possible. For example, we have used a "delayed match-to-location" task in which the rat is shown a marked goal in the first half of each trial and then must go to the same place with the goal unmarked on the second half of each trial. Normal or sham lesioned rats readily learn this variant, whereas rats with extensive hippocampal lesions show virtually no improvement in performance after many trials (Kubie \& Muller, unpublished results).

If the water maze and the dry maze are so similar, why design a new task? The original reason was straightforward: We intended to do place cell recordings during performance of a hidden-goal task and believed it would be easier without the need to seal the electrical system. We soon realized, however, that the dry maze itself is valuable as a hidden-goal navigational task.

One nice property of the dry maze is purely practical: It is inexpensive to build and maintain. For instance, the water in a tank $2 \mathrm{~m}$ in diameter and $0.5 \mathrm{~m}$ deep weighs about $1,600 \mathrm{~kg}$. It is a significant problem to keep this 
much water at a fixed temperature, to change it when necessary, and so on. In contrast, the dry maze requires only $30-50 \mathrm{~kg}$ of sawdust, which can be freed of feces by raking and be easily replaced.

The fact that the dry maze is an appetitive task rather than an escape task is also useful since it is then easier to design experiments in which food is buried at several places. In this way, it is possible to test the memory limits of the animal, to determine whether its solutions to this version of the traveling salesman problem are efficient, and so on. We have successfully trained rats to go successively to three locations (Chapuis, Kubie, \& Muller, unpublished observations). In principle, a swimming task with multiple, retractable platforms could be used, but the appetitive version is much simpler.

Another valuable property of the dry maze is that the rat must perform a specific behavior-digging at the goal location-to solve the problem. The rat may dig at several locations before it finds the correct one, revealing a hierarchy of hypothesized goal locations. In contrast, in the swimming task, the rat swims until it encounters the hidden platform, at which point the problem is already solved except for climbing. Only when the platform is not present at an expected location does the rat engage in behavior that directly indicates its hypothesis.

A final useful quality of the dry maze is that the food is rarely if ever found by random, repetitive digging. This strategy is so inefficient that rats cease to dig at all when they are consistently unable to locate the food with a directed search. The great difficulty of finding the buried food accidentally or by random search stands in contrast to the virtual certainty that random swimming or even a circling strategy will relatively rapidly cause the rat to encounter the platform. In consequence, there is little of the "floor" effect often seen in the swimming task, in which the latency difference between poor and good performance is quite small. Thus, it is possible to quantify performance in the dry maze using latency or corrected path length (observed path - shortest path) instead of having to resort to quadrant search time in probe trials.

The marked increase in latency seen immediately after either 1- or 14-week training-surgery intervals for sham lesioned rats is in contrast to the usual good retention in the swimming task (Schallert, Day, Weisend, \& Sutherland, 1996). This effect probably arises from two causes. First, we reported the latency (averaged across rats) for each trial rather than for blocks of four trials, as is often done in work on the swimming task. For this reason, the apparent gradient for relearning is steeper. A more important reason may concern the motivational state of the animal. In the buried food task, the rat is in no danger during a trial. It may therefore be that the reduced performance reveals a tendency for reexploration rather than forgetting per se. It would therefore be interesting to look at the rate of relearning as a function of the duration of food deprivation before testing; if the main issue is mo- tivation, longer periods of deprivation should lead to better initial performance and a faster approach to asymptotic performance.

\section{Hippocampal Lesions and \\ Amnesia for Navigational Tasks}

Why do we see graded retrograde amnesia in the dry maze task when most experiments with the swimming task find grave performance deficits at all traininglesion intervals? One possibility is that the dry maze task is more difficult. The swimming task can be solved with a wide variety of strategies, including swimming a certain distance from the wall or quartering the pool. Lesioned rats even adopt sophisticated strategies in which they make loops while swimming toward the hidden platform, a strategy similar to groping in the dark (Whishaw $\&$ Tomie, 1997a). Thus, useful strategies for finding the hidden platform are available without relying on the vector field representation. In contrast, the food cannot be found in the dry maze task without specific information about its location. Thus, according to our argument, the rats are forced to use the vector field representation. This necessarily results in short latencies because the vector field representation stores precisely that information necessary to take the shortest path to the goal and no other information. In this view, it would be possible to improve performance in the swimming task by making it more difficult or more important to find the platform quickly, perhaps by increasing the size of the pool or by decreasing the temperature of the water.

A second possible explanation of why we saw graded retrograde amnesia is that the sensory stimuli available in the dry maze task are more restricted than those in the swimming task. It might be that the simplicity of the cues in the dry maze cylinder makes it easier to build a vector field representation. In this case, reducing the stimulus complexity in the swimming task might result in graded retrograde amnesia, and adding distal cue complexity in the dry maze task might reduce performance at long training-lesion intervals.

Recent experiments show that naive rats with hippocampal lesions can subsequently learn to get to a hidden platform in the swimming task if the problem is initially very easy and is made harder in small steps (Schallert et al., 1996; Whishaw, Cassel, \& Jarrad, 1995). Perhaps procedures of this kind make it possible to establish a vector field representation by enabling the animal to consistently take straight paths to the goal, thereby eliminating competing, easier responses. In this regard, it is interesting that Whishaw and Tomie (1997b) found that lesioned rats that learned to go to a first goal location could not find a new goal location even though they moved directly to that location on the one out of every four trials in which the hidden platform was replaced with a visible platform. This result reinforces the idea that the proposed neocortical vector field representation can function indepen- 
dently of the hippocampus, but that hippocampal circuitry is extremely important for either changing vector directions to point to a new goal or to establish a second or third vector field representation to be used under altered behavioral circumstances.

Our results are also related in a general way to a recent swimming experiment by Pearce, Roberts, and Good (1998), who showed that rats with hippocampal lesions can locate a goal platform relative to a landmark in the pool. They saw that hippocampally damaged rats adopt a beacon-like strategy more rapidly than normal animals; the damaged rats swim to the vicinity of the landmark regardless of its placement on earlier trials than do normal rats. The strategy is not, however, to swim directly to the landmark and then initiate a random search. Instead, by moving the platform relative to the landmark on a probe trial, Pearce et al. showed that hippocampally lesioned rats have information about the direction between the landmark and the platform. This strategy may reflect the use of a head direction system (see Muller, Ranck, \& Taube, 1996 , for a recent review) or other navigational capacities. The ability to use this navigation-like strategy is, however, an independent demonstration that the hippocampus is not the sole repository of sophisticated spatial behavior, even though it appears necessary for the entire range of mapping capacities.

\section{REFERENCES}

Angeli, S. J., Murray, E. A., \& Mishkin, M. (1993). Hippocampectomized monkeys can remember one place but not two. Neumpsychologia, 31, 1021-1030.

Astur, R. S., Mumby, D. G., Weisend, M. P., \& Sutherland, R. J. (1994). Hippocampal damage in rats causes retrograde amnesia for place navigation but not object discriminations. Society for Neuroscience Abstracts, 20, 1015

BLUм, K. I., \& Аввотт, L. F. (1996). A model of spatial map formation in the hippocampus of the rat. Neural Computation, 8, 85-93.

Bolmuis, J. J., Stewarr, C. A., \& Forrest, E. M. (1994). Retrograde amnesia and memory reactivation in rats with ibotenate lesions to the hippocampus or subiculum. Quarterly Journal of Experimental Psychology, 47B, 129-150.

Bostock, E., Muller, R. U., \& Kubie, J. L. (1991). Experiencedependent modifications of hippocampal place cell firing. Hippocampus, 1, 193-206.

CHO, Y. H., \& KeSNER, R. P. (1996). Involvement of entorhinal cortex or parietal cortex in long-term spatial discrimination memory in rats: Retrograde amnesia. Behavioral Neuroscience, 110, 436-442.

Cohen, N. J., \& Eichenbaum, H. (1993). Memory, amnesia, and the hippocampal system. Cambridge, MA: MIT Press.

Corkin, S. (1965). Tactually-guided maze learning in man: Effects of unilateral cortical excisions and bilateral hippocampal lesions. Neuropsychologia, 3, 339-351.

CORKIN, S. (1968). Acquisition of motor skill after bilateral medial temporal lobe excision. Neuropsychologia, 6, 255-265.

GERSTNER, W., \& ABBotT, L. F. (1997). Learning navigational maps through potentiation and modulation of hippocampal place cells. Journal of Computational Neuroscience, 4, 79-94.

JARRARD, L. E., \& MELDRUM, B. S. (1993). Selective excitotoxic pathology in the rat hippocampus. Neuropathology \& Applied Neurobiology, 19, 381-389.

KIM, J. J., \& FANSELOW, M. S. (1992). Modalilty-specific retrograde amnesia of fear. Science, 22, 675-677.

Kubie, J. L., Muller, R. U., Dayyani, S., \& Morgenstern, D. (1987).
Behavioral responses parallel the responses of hippocampal neurons to environmental manipulations. Society for Neuroscience Abstracts, $13,1068$.

McClelland, J. L., McNaughton, B. L., \& O'Reilly, R. C. (1995). Why there are complementary learning systems in the hippocampus and neocortex: Insights from the successes and failures of connectionist models of learning and memory. Psychological Review, 102, 419-457.

Meunier, M., Bachevalier, J., Mishkin, M., \& Murray, E. A. (1993). Effects on visual recognition of combined and separate ablations of the entorhinal and perirhinal cortex in rhesus monkeys. Journal of Neumscience, 13, 5418-5432.

Meunier, M., Hadfield, W., Bachevalier, J., \& Murray, E. A. (1996). Effects of rhinal cortex lesions combined with hippocampectomy on visual recognition memory in rhesus monkey. Journal of Neurophysiology, 75, 1190-1205.

MORRIS, R. G. M. (1984). Developments of a water-maze procedure for studying spatial learning in the rat. Journal of Neuroscience Methods, 11, 47-60.

Morris, R. G. M., Garrud, P., Rawlins, J. N. P., \& O'Keefe, J. (1982). Place navigation impaired in rats with hippocampal lesions. Nature, 297, 681-683.

Morris, R. G. M., Schenk, F., Tweedie, F., \& Jarrard, L. E. (1990). Ibotenate lesions of hippocampus and/or subiculum: Dissociating components of allocentric spatial learning. European Journal of Neuroscience, 2, 1016-1028.

MULler, R. U. (1996). A quarter century of place cells. Neuron, 17, 120.

Muller, R. U., \& KubIE, J. L. (1987). The effects of changes in the environment on the spatial firing of hippocampal complex-spike cells. Journal of Neuroscience, 7, 1951-1968.

Muller, R. U., KuBIE, J. L., \& SAYPofF, R. (1991). The hippocampus as a cognitive graph (abridged version). Hippocampus, 1, 243-246.

Muller, R. U., Ranck, J. B., \& TAube, J. S. (1996). Head direction cells--Properties and functional significance. Current Opinion in Neurobiology, 6, 196-206.

Muller, R. U., Stead, M., \& PACH, J. (1996). The hippocampus as a cognitive graph. Journal of General Physiology, 107, 663-694.

Mumby, D. G., Wood, E. R., \& Pinel, P. J. (1992). Object-recognition memory is only mildly impaired in rats with lesions of hippocampus and amygdala. Psychobiology, 20, 18-27.

NadEL, L. (1991). The hippocampus and space revisited. Hippocampus, 1, 221-229.

NADEL, L., \& Moscovitch, M. (1997). Memory consolidation, retrograde amnesia and the hippocampal complex. Current Opinion in Neurobiology, 7, 217-227.

O'KeEFE, J., \& NADEL, L. (1978). The hippocampus as a cognitive map London: Clarendon Press.

PAXINOS, G. (1986). The rat brain brain in stereotaxic coordinates. New York: Academic Press.

Pearce, J. M., Roberts, A. D. L., \& Good, M. (1998). Hippocampal lesions disrupt navigation based on cognitive maps but not heading vectors. Nature, 396, 75-77.

Schallert, T., Day, L. B., Weisend, M., \& Sutherland, R. J. (1996). Spatial learning by hippocampal rats in the Morris water task. Society for Neuroscience Abstracts, 22, 678.

Squire, L. R., \& Alvarez, P. (1995). Retrograde amnesia and memory consolidation: A neurobiological perspective. Current Opinion in Neurobiology, 5, 169-177.

Sutherland, R. J., Arnold, K. A., \& Rodriguez, A. R. (1987). Anterograde and retrograde effects on place memory after limbic or diencephalic damage. Society for Neuroscience Abstracts, 13, 1066.

Sutherland, R. J., WhishaW, I. Q., \& Kolb, B. (1983). A behavioural analysis of spatial localization following electrolytic, kainate- or colchicine-induced damage to the hippocampal formation in the rat. Behavioural Brain Research, 7, 133-153.

ThOMPSON, L. T., \& BEST, P. J. (1990). Long-term stability of the placefield activity of single units recorded from the dorsal hippocampus of freely behaving rats. Brain Research, 509, 299-308.

VNeK, N., \& RothbLaTt, L. A. (1996). The hippocampus and long- 
term object memory in the rat. Journal of Neuroscience, 16, 27802787.

Whishaw, I. Q., CASSEl, J. C., \& JARRAD, L. E. (1995). Rats with fimbriafornix lesions display a place response in a swimming pool: Dissociation between getting there and knowing where. Journal of Neuroscience, $\mathbf{1 5}, 5779$.

Whishaw, I. Q., RoD, M. R., \& AuER, R. N. (1994). Behavioral deficits revealed by multiple tests in rats with ischemic damage limited to half of the CAl sector of the hippocampus. Brain Research Bulletin, 34, 383-389.

Whishaw, I. Q., \& Tomie, J. A. (1997a). Perseveration on place reversals in spatial swimming pool tasks: Further evidence for place learning in hippocampal rats. Hippocampus, 7, 361-370.

Whishaw, I. Q., \& Tomie, J. A. (1997b). Piloting and dead reckoning dissociated by fimbria-fornix lesions in a rat food carrying task. Behavioural Brain Research, 89, 87-97.
Wible, C. G., Shiber, J. R., \& Olton, D. S. (1992). Hippocampus, fimbria-fornix, amygdala, and memory: Object discriminations in rats. Behavioral Neuroscience, 106, 751-761.

WINOCUR, G. (1990). Anterograde and retrograde amnesia in rats with dorsal hippocampal or dorsomedial thalamic lesions. Behavioural Brain Research, 38, 145-154.

\section{NOTE}

1. Subsequent work by Gerstner and Abbott (1997) shows how the vector field scheme can be elaborated to permit two or more goals to be represented in a single environment, but the original single-goal model fits our data nicely.

(Manuscript received November 2, 1998; revision accepted for publication March 19, 1999.) 